PROCEEDINGS OF THE

AMERICAN MATHEMATICAL SOCIETY

Volume 134, Number 9, September 2006, Pages 2511-2520

S 0002-9939(06)08515-7

Article electronically published on February 17, 2006

\title{
HODGE STRUCTURES FOR ORBIFOLD COHOMOLOGY
}

\author{
JAVIER FERNANDEZ
}

(Communicated by Michael Stillman)

\begin{abstract}
We construct a polarized Hodge structure on the primitive part of Chen and Ruan's orbifold cohomology $H_{o r b}^{k}(X)$ for projective $S L$-orbifolds $X$ satisfying a "Hard Lefschetz Condition". Furthermore, the total cohomology $\bigoplus_{p, q} H_{o r b}^{p, q}(X)$ forms a mixed Hodge structure that is polarized by every element of the Kähler cone of $X$. Using results of Cattani-Kaplan-Schmid this implies the existence of an abstract polarized variation of Hodge structure on the complexified Kähler cone of $X$.

This construction should be considered as the analogue of the abstract polarized variation of Hodge structure that can be attached to the singular cohomology of a crepant resolution of $X$, in light of the conjectural correspondence between the (quantum) orbifold cohomology and the (quantum) cohomology of a crepant resolution.
\end{abstract}

\section{INTRODUCTION}

The cohomology of a projective variety $X$ underlies several very rich algebraic structures. For example, if $X$ is smooth, the Hodge decomposition of $H^{k}(X, \mathbb{R})$ defines a pure Hodge structure that is, in fact, polarized when restricted to its primitive part. In general, a result of P. Deligne constructs a mixed Hodge structure over $H^{k}(X, \mathbb{R})$. Also, the total cohomology of a smooth projective variety defines a mixed Hodge structure polarized by any Kähler class of $X$. A result of J. Steenbrink and M. Saito proves that this is still true for projective orbifolds.

Motivated by some ideas that originated in physics, W. Chen and Y. Ruan defined a new cohomology theory for orbifolds: the orbifold cohomology. The purpose of this note is to explore under what conditions the total orbifold cohomology of a projective orbifold has a Hodge structure similar to that of a smooth projective variety. Our main result is that if $X$ is a projective $S L$-orbifold satisfying a certain condition on the ages of its local groups (that we call the Hard Lefschetz Condition), then $H_{\text {orb }}^{*}(X, \mathbb{R})$ underlies a mixed Hodge structure that is polarized by any Kähler class. This result will follow from the Hard Lefschetz Theorem, the Lefschetz decomposition and the Hodge-Riemann bilinear relations, all of which will be seen to hold. As a consequence of this result and the work of E. Cattani, A. Kaplan and W. Schmid, we also conclude that under the previous conditions there is a natural polarized variation of Hodge structures defined over the complexified

Received by the editors May 31, 2004 and, in revised form, March 29, 2005.

2000 Mathematics Subject Classification. Primary 14F43, 14C30; Secondary 14J32.

Key words and phrases. Orbifold cohomology, polarized Hodge structure, Lefschetz package.

(C)2006 American Mathematical Society

Reverts to public domain 28 years from publication 2511 
Kähler cone of $X$. This variation is the asymptotic approximation of a conjectural $A$-model variation of Hodge structure [8, 2, for Calabi-Yau orbifolds.

Our results show that there is a close similarity between the Hodge structures on the orbifold cohomology of a projective orbifold satisfying the Hard Lefschetz Condition and the standard cohomology of a smooth projective variety. This similarity can be considered as indirect evidence for the several conjectures posed by Ruan [11] regarding the existence of equivalences between the orbifold cohomology of an orbifold $X$ and the cohomology of a crepant resolution of $X$.

We will review some notions of Hodge theory and orbifold cohomology in Sections 2 and 3. The Hard Lefschetz Theorem is discussed in Section 4, while Section 5 analyzes the polarized Hodge structure on the orbifold cohomology of appropriate orbifolds.

\section{Hodge THEORY PRELIMINARIES}

In this section we recall the basic notions of Hodge theory. Additionally, we state some classical results for the de Rham and Dolbeault cohomologies of a projective orbifold. We refer to [4, 5, 9, 15, for more details and proofs.

Let $V$ be a finite dimensional $\mathbb{R}$-vector space and $k \in \mathbb{Z}$. A (real) Hodge structure of weight $k$ on $V$ is a decomposition of $V_{\mathbb{C}}:=\mathbb{C} \otimes V, V_{\mathbb{C}}=\bigoplus_{p} H^{p, k-p}$ such that $\overline{H^{p, k-p}}=H^{k-p, p}$ for all $p$. Alternatively, such Hodge structure is described by a decreasing filtration $F$ of $V_{\mathbb{C}}$ such that $V_{\mathbb{C}}=F^{p} \oplus \overline{F^{k-p+1}}$ for all $p$. The relation between $F$ and $H$ is that $F^{p}=\bigoplus_{a>p} H^{a, k-a}$ while $H^{a, k-a}=F^{a} \cap \overline{F^{k-a}}$. The numbers $h^{p, q}:=\operatorname{dim} H^{p, q}$ are called the Hodge numbers of the structure.

A Hodge structure $(V, H, k)$ is polarized by the nondegenerate, bilinear, $(-1)^{k}$ symmetric form $Q$ on $V$ if

$$
\begin{gathered}
Q\left(H^{p, k-p}, H^{q, k-q}\right)=0, \quad \text { unless } p+q=k, \\
Q(C v, \bar{v})>0, \quad \text { for all } v \in V_{\mathbb{C}}-\{0\},
\end{gathered}
$$

where $C: V_{\mathbb{C}} \rightarrow V_{\mathbb{C}}$ is the Weil operator defined by $C(v):=i^{p-q} v$ for $v \in H^{p, q}$.

Example 2.1. For a smooth projective or compact Kähler manifold $X$ of dimension $n$ with a choice of Kähler form $\omega$ and $k=0, \ldots, n$, the primitive part of the cohomology $H_{o}^{k}(X, \mathbb{C})($ see $(2.5))$ is a Hodge structure of weight $k$ polarized by the form

$$
Q_{k}(\alpha, \beta):=Q\left(\alpha, \beta \wedge \omega^{n-k}\right), \quad \text { if } \alpha, \beta \in H^{k}(X, \mathbb{C}),
$$

where

$$
Q(\alpha, \beta):=(-1)^{k(k-1) / 2} \int_{X} \alpha \wedge \beta, \quad \text { for } \alpha \in H^{k}(X, \mathbb{C}) .
$$

The primitive cohomology of a smooth projective variety $X$ is the typical example of a polarized Hodge structure. The same properties can be extended to the case where $X$ has some mild singularities. This is the case when $X$ is a projective orbifold, that is, an orbifold that can be realized as a projective variety. In the following, we will be mostly interested in this setup. We refer to [14, 7, 6] for the notion of orbifold.

Let $X$ be a projective orbifold of dimension $n$ with Kähler class $\omega \in H^{1,1}(X, \mathbb{R}):=$ $H^{1,1}(X) \cap H^{2}(X, \mathbb{R})$. Define

$$
L_{\omega}: H^{*}(X, \mathbb{R}) \rightarrow H^{*}(X, \mathbb{R}), \quad L_{\omega}(\alpha):=\omega \wedge \alpha .
$$


The primitive cohomology of $X$ is defined as

$$
H_{o}^{p}(X, \mathbb{C}):=\operatorname{ker}\left(L_{\omega}^{n-p+1}: H^{p}(X, \mathbb{C}) \rightarrow H^{2 n-p+2}(X, \mathbb{C})\right)
$$

for $p=0, \ldots, n$ and $\{0\}$ otherwise.

Theorem 2.2. Let $X$ be a projective orbifold of dimension $n$ with Kähler class $\omega \in H^{1,1}(X, \mathbb{R})$.

(1) (Hard Lefschetz) For all $p \in \mathbb{N}$ the map $L_{\omega}^{p}$ induces an isomorphism between $H^{n-p}(X, \mathbb{R})$ and $H^{n+p}(X, \mathbb{R})$.

(2) (Lefschetz decomposition) For $k=0, \ldots, n$ :

$$
H^{k}(X, \mathbb{C})=\bigoplus_{p \geq 0} L_{\omega}^{p} H_{o}^{k-2 p}(X, \mathbb{C}) .
$$

(3) For all $k=0, \ldots, 2 n$ there is a decomposition

$$
H^{k}(X, \mathbb{C})=\bigoplus_{p} H^{p, k-p}(X) .
$$

For $k=0, \ldots, n$, 2.7) restricts to the primitive cohomology to give

$$
H_{o}^{k}(X, \mathbb{C})=\bigoplus_{p} H_{o}^{p, k-p}(X)
$$

where $H_{o}^{p, k-p}(X)=H^{p, k-p}(X) \cap H_{o}^{k}(X, \mathbb{C})$. Both decompositions (2.7) and (2.8) define Hodge structures of weight $k$ on the underlying $H^{k}(X, \mathbb{R})$ and $H_{o}^{k}(X, \mathbb{R})$ respectively.

(4) The Hodge structure on the primitive cohomology (2.8) is polarized by the form (2.3).

Theorem 2.2 is due to J. Steenbrink [16, §1], completed by M. Saito [13]. In Section 5 we will derive the analogue of Theorem 2.2 for the orbifold cohomology.

A mixed Hodge Structure (MHS) on $V$ consists of a pair of filtrations of $V_{\mathbb{C}}$, $(W, F), W$ defined over $\mathbb{R}$ and increasing, $F$ decreasing, such that $F$ induces a Hodge structure of weight $p$ on $\mathrm{Gr}_{p}^{W}$ for each $p$. Equivalently, an MHS on $V$ is a bigrading $V_{\mathbb{C}}=\bigoplus I^{p, q}$ satisfying $I^{p, q} \equiv \overline{I^{q, p}} \bmod \left(\bigoplus_{a<p, b<q} I^{a, b}\right)$ (see [5, Theorem 2.13]). Given such a bigrading we define: $W_{l}=\bigoplus_{p+q \leq l} I^{p, q}, F^{a}=\bigoplus_{p \geq a} I^{p, q}$. An MHS is said to split over $\mathbb{R}$ if $I^{p, q}=\overline{I^{q, p}}$. A map $T \in \mathfrak{g l}\left(V_{\mathbb{C}}\right)$ such that $T\left(I^{p, q}\right) \subset I^{p+a, q+b}$ is called a morphism of bidegree $(a, b)$.

A nilpotent linear transformation $N \in \mathfrak{g l}(V)$ defines an increasing filtration, the weight filtration, $W(N)$ of $V$, uniquely characterized by requiring that, for all $l$, $N\left(W_{l}(N)\right) \subset W_{l-2}(N)$ and that $N^{l}: \mathrm{Gr}_{l}^{W(N)} \rightarrow \mathrm{Gr}_{-l}^{W(N)}$ be an isomorphism.

Definition 2.3. A polarized mixed Hodge structure (PMHS) [3, (2.4)] of weight $k$ on $V$ consists of an MHS $(W, F)$ on $V$, a nondegenerate, bilinear, $(-1)^{k}$-symmetric form $Q$, and a $(-1,-1)$-morphism $N \in \mathfrak{g}_{\mathbb{R}}$, the Lie algebra of $O(V, Q)$, such that

(1) $N^{k+1}=0$

(2) $W=W(N)[-k]$, where $W[-k]_{j}=W_{j-k}$,

(3) $Q\left(F^{a}, F^{k-a+1}\right)=0$, and

(4) the Hodge structure of weight $k+l$ induced by $F$ on $\operatorname{ker}\left(N^{l+1}: \operatorname{Gr}_{k+l}^{W} \rightarrow\right.$ $\left.\mathrm{Gr}_{k-l-2}^{W}\right)$ is polarized by $Q\left(\cdot, N^{l} \cdot\right)$. 
Polarized MHSs arise naturally as limits of polarized variations of Hodge structure. Conversely, a PMHS generates a nilpotent orbit. Indeed, there is an equivalence between nilpotent orbits and mixed Hodge structures polarized by an abelian family of operators as explained by Theorem (2.3) in [4].

Example 2.4. Let $X$ be an $n$-dimensional, smooth projective variety. Let $V=$ $H^{*}(X, \mathbb{R})$. The bigrading $I^{p, q}:=H^{n-q, n-p}(X)$ defines an MHS on $V$ which splits over $\mathbb{R}$. The weight and Hodge filtrations are then

$$
W_{l}=\bigoplus_{d \geq 2 n-l} H^{d}(X, \mathbb{C}), \quad F^{p}=\bigoplus_{r} \bigoplus_{s \leq n-p} H^{r, s}(X) .
$$

Given a Kähler class $\omega \in H^{1,1}(X, \mathbb{R})$, let $L_{\omega} \in \mathfrak{g r}\left(V_{\mathbb{R}}\right)$ denote multiplication by $\omega$. Note that $L_{\omega}$ is an infinitesimal automorphism of the form $Q$ defined in (2.4) and is a $(-1,-1)$ morphism of $(W, F)$. Moreover, the Hard Lefschetz Theorem and the Riemann bilinear relations are equivalent to the assertion that $L_{\omega}$ together with $Q$ polarize $(W, F)$. Let $\mathcal{K} \subset H^{1,1}(X, \mathbb{R})$ denote the Kähler cone and

$$
\mathcal{K}_{\mathbb{C}}:=H^{1,1}(X, \mathbb{R}) \oplus i \mathcal{K} \subset H^{2}(X, \mathbb{C})
$$

the complexified Kähler cone. It then follows from Theorem (2.3) in 4 that for every $\xi \in \mathcal{K}_{\mathbb{C}}$, the filtration $\exp \left(L_{\xi}\right) \cdot F$ is a Hodge structure of weight $n$ on $V$ polarized by $Q$. The map $\xi \in \mathcal{K}_{\mathbb{C}} \mapsto \exp \left(L_{\xi}\right) \cdot F$ is the period map (in fact, the nilpotent orbit) of a variation of Hodge structure over $\mathcal{K}_{\mathbb{C}}$.

Theorem 5.3 and Corollary 5.4 in Section 5 will prove similar properties for the orbifold cohomology.

\section{ORBIFOLD COHOMOLOGY}

In this section we briefly review Chen and Ruan's construction of orbifold cohomology [].

Recall that to an orbifold $X$ we can associate another orbifold, known as the inertia orbifold

$$
\tilde{X}:=\left\{(p,(g)): p \in X, g \in G_{p}\right\}
$$

where $(g)$ denotes the conjugacy class of $g$ in $G_{p}$, the local group of $X$ at $p$. If $\left\{\left(V_{p}, G_{p}, \pi_{p}\right): p \in X\right\}$ is a uniformizing system for $X$, a uniformizing system for $\tilde{X}$ is $\left\{\left(V_{p}^{g}, C(g), \pi_{p, g}\right):(p,(g)) \in \tilde{X}\right\}$, where $V_{p}^{g}$ is the fixed point set of $g$, and $C(g)$ is the centralizer of $g$ in $G_{p}$. The topology on $\tilde{X}$ is defined so that the sets $\pi_{p, g}\left(V_{p}^{g}\right) \simeq V_{p}^{g} / C(g)$ are open.

Even if $X$ is a connected space, $\tilde{X}$ need not be. In general, $\tilde{X}$ decomposes in connected components

$$
\tilde{X}=\bigsqcup_{t \in T} X_{t}
$$

where $t$ labels each component and $T$ is the set of all such labels. The components are orbifolds that are compact (complex) if $X$ is compact (complex). Also, if $X$ is projective, so are the components (use [1]).

We denote by $T^{0} \subset T$ the set of connected components that contain points of the form $(p,(1))$, where $1 \in G_{p}$ is the identity. Also, $X_{1}:=\bigsqcup_{t \in T^{0}} X_{t} . X_{1}$ is called the nontwisted sector, while the other connected components of $\tilde{X}$ are the twisted sectors. 
Define $\pi: \tilde{X} \rightarrow X$ by $\pi(p,(g)):=p$ and let $\pi_{t}$ denote the restriction of $\pi$ to the sector $X_{t}$. Note that $\pi_{1}: X_{1} \rightarrow X$ is an isomorphism; hence, when $X$ is connected, so is $X_{1}$. Also, define the involution $I: \tilde{X} \rightarrow \tilde{X}$ by $I(p,(g)):=\left(p,\left(g^{-1}\right)\right)$. We also denote by $I$ the involution induced on $T$.

For each $g \in G_{p}$ we consider the action $\rho_{p}(g)$ induced by $g$ on $T_{p} X$. The eigenvalues of $\rho_{p}(g)$ are of the form $\exp \left(2 \pi i \frac{m_{j}}{m_{g}}\right)$, where $m_{g}$ is the order of $g$ and $m_{j} \in \mathbb{Z}, 0 \leq m_{j}<m_{g}$. Then, the index of $g$ at $p$ (also known as the age or degree shifting number of $g$ ) is defined by

$$
i_{(p, g)}:=\sum_{j=1}^{n} \frac{m_{j}}{m_{g}} \in \mathbb{Q} \quad \text { where } \quad n:=\operatorname{dim}_{\mathbb{C}} X .
$$

This index defines a locally constant function of $p$. If $X_{t}$ is the sector containing $(p,(g))$, we define $i_{t}:=i_{(p, g)}$. Then [7, Lemma 3.2.1] shows that $i_{(p, g)}$ is integral if and only if $\rho_{p}(g) \in S L(n, \mathbb{C})$ and that the (complex) dimension of $X_{t}$ is

$$
\operatorname{dim}_{\mathbb{C}} X_{t}=\operatorname{dim}_{\mathbb{C}} X-i_{t}-i_{I(t)}
$$

An orbifold for which $\rho_{p}(g) \in S L(n, \mathbb{C})$ for all $p$ and $g$ is called an $S L$-orbifold. In algebro-geometric terms, this is to say that (the geometric space associated to) $X$ is a Gorenstein variety. In particular, for instance, if $X$ is Calabi-Yau, then $X$ is an $S L$-orbifold.

The orbifold cohomology groups of the orbifold $X$ are defined by

$$
H_{\text {orb }}^{k}(X, \mathbb{R}):=\bigoplus_{t \in T} H^{k-2 i_{t}}\left(X_{t}, \mathbb{R}\right)
$$

where the cohomology groups on the right are the singular cohomology groups with real coefficients, which are isomorphic to the corresponding de Rham cohomology groups [14. If $X$ is complex, then the orbifold Dolbeault cohomology groups are defined by

$$
H_{o r b}^{p, q}(X):=\bigoplus_{t \in T} H^{p-i_{t}, q-i_{t}}\left(X_{t}\right)
$$

Chen and Ruan define a product structure on orbifold cohomology. We will not go into all the details of this definition for which we refer, once more, to [7. The orbifold product defines a rich structure, as the following result shows [7, Theorems 4.1.5 and 4.1.7].

Theorem 3.1. Let $X$ be a compact, complex orbifold. Then the orbifold product is bigraded,

$$
\smile_{o r b}: H_{o r b}^{p, q}(X) \times H_{o r b}^{p^{\prime}, q^{\prime}}(X) \rightarrow H_{o r b}^{p+p^{\prime}, q+q^{\prime}}(X) .
$$

The total cohomologies $H_{o r b}^{*}(X)$ and $H_{\text {orb }}^{*, *}(X)$ become rings under $\smile_{\text {orb }}$, with unit $e_{0}^{X} \in H_{o r b}^{0,0}(X)$. When $X$ is an $S L$-orbifold both rings are supercommutative. Finally, the restriction of $\smile_{\text {orb }}$ to the nontwisted sector coincides with the cup product on $H^{*}\left(X_{1}\right)$ and $H^{*, *}\left(X_{1}\right)$.

The cohomology of the twisted sector $X_{t}, H^{*}\left(X_{t}\right)$, becomes an $H^{*}\left(X_{1}\right)$-module under $\smile_{o r b}$. The following result relates this module structure to the standard cup product on $H^{*}\left(X_{t}\right)$, and will be useful to study the left (orbifold) multiplication by Kähler classes.

Proposition 3.2. Let $X$ be a compact orbifold, $\alpha \in H^{p}\left(X_{1}\right)$ and $\beta \in H^{q}\left(X_{t}\right)$ for $t \in T$. Then $\alpha \smile_{\text {orb }} \beta=\left(\pi_{1}^{-1} \circ \pi_{t}\right)^{*}(\alpha) \wedge \beta \in H^{p+q}\left(X_{t}\right)$. 
Proof. In the language of [7, §4], the obstruction bundle is trivial for dimensional reasons and the proposition follows.

\section{The Hard Lefschetz Condition}

In this section we want to find a necessary and sufficient condition for the Hard Lefschetz Theorem to hold on the orbifold cohomology.

As we mentioned in the Introduction, it is generally believed that, under some yet not well understood conditions, there should be some kind of equivalence between the orbifold cohomology of an orbifold $X$ and the cohomology of a crepant resolution of $X$. For instance, if $Y \rightarrow X$ is a hyperkähler resolution, Ruan conjectured such a relation [12, 11]. In any case, if such equivalence exists, the algebraic structure of the cohomology of the resolution should have an analogue in the orbifold cohomology of $X$. One "feature" of the singular cohomology of the resolution is the Hard Lefschetz Theorem. We will concentrate here on the analogous result for the orbifold cohomology.

If $X$ is a Kähler orbifold, for any Kähler class $\omega \in H^{1,1}(X, \mathbb{R})$ we define the operator

$$
L_{\omega}: H_{\text {orb }}^{*}(X) \rightarrow H_{\text {orb }}^{*}(X), \quad L_{\omega}(\alpha):=\pi_{1}^{*} \omega \smile_{\text {orb }} \alpha .
$$

As we mentioned in Section 2, the Hard Lefschetz Theorem states that for any Kähler class $\omega$ on the projective orbifold $X$ of complex dimension $n$, for all $p \in \mathbb{N}$ the map $L_{\omega}^{p}$ induces an isomorphism between $H_{\text {orb }}^{n-p}(X, \mathbb{R})$ and $H_{\text {orb }}^{n+p}(X, \mathbb{R})$. To understand this condition, we remember that the orbifold product by $\omega^{p} \in$ $H^{p, p}(X) \simeq H^{p, p}\left(X_{1}\right)$ preserves the forms on each sector, that is, for all $t \in T$, $L_{\omega}^{p}\left(H^{*}\left(X_{t}\right)\right) \subset H^{*}\left(X_{t}\right)$. Then if $L_{\omega}^{p}$ is an isomorphism, it should induce isomorphisms on each $H^{*}\left(X_{t}\right)$. But care must be taken regarding the degrees: $L_{\omega}^{p}$ pairs $H_{\text {orb }}^{n-p}(X, \mathbb{R})$ and $H_{\text {orb }}^{n+p}(X, \mathbb{R})$. So, for each $t, L_{\omega}^{p}$ is pairing $H^{n-p-2 i_{t}}\left(X_{t}\right)$ with $H^{n+p-2 i_{t}}\left(X_{t}\right)$. It is easy to check using (3.1) that this is not possible unless

$$
i_{t}=i_{I(t)} \quad \text { for all } t \in T \text {. }
$$

Finally, it follows immediately from Theorem 2.2 that when (4.2) holds, $L_{\omega}^{p}$ are isomorphisms for all $p$. Thus we have proved the following.

Proposition 4.1. Let $X$ be a projective orbifold of dimension $n$ that satisfies the Hard Lefschetz Condition (4.2). Then for each Kähler class $\omega$ on X, the operator

$$
L_{\omega}^{p}: H_{\text {orb }}^{n-p}(X, \mathbb{R}) \rightarrow H_{\text {orb }}^{n+p}(X, \mathbb{R})
$$

is an isomorphism.

Remark 4.2. Condition (4.2) had already appeared in [11, §4] in connection with the possibility of defining a "hermitian" inner product to fix a signature problem in the Kummer surface example.

Remark 4.3. Condition (4.2) holds trivially for orbifolds of dimension 2 and for orbifolds whose nontrivial local groups are isomorphic to $\mathbb{Z}_{2}$.

Example 4.4. Let $\mathbb{P}_{\Delta}$ be the simplicial complete Fano toric variety of dimension $n$ associated to the reflexive polytope $\Delta \subset \mathbb{R}^{n}$. If $X$ is a generic anticanonical hypersurface of $P$, then $X$ is a reduced Calabi-Yau projective orbifold. M. Poddar shows in [10, §4.2] that the twisted sectors of $X$ are isomorphic to $X \cap \overline{O_{\tau}}$, where $\overline{O_{\tau}}$ is the closure of the torus orbit in $\mathbb{P}_{\Delta}$ associated to the cone $\tau$ obtained as the 
cone over a face $F^{0}$ of $\Delta^{0}$, the polar polytope of $\Delta$, and where $1 \leq \operatorname{dim} F^{0} \leq n-2$ (it is easy to see that $\left.\operatorname{dim} \overline{O_{\tau}}=n-\left(1+\operatorname{dim} F^{0}\right)\right)$. Also, it is shown that there is, at least, one twisted sector $X_{t}$ of the previous form with $i_{t}=1$ for each lattice point in the relative interior of $F^{0}$ (actually the result is more specific, but that is not required in our application).

For example, the weighted projective space $\mathbb{P}(1,1,2,2,6)$ is $\mathbb{P}_{\Delta}$ where $\Delta$ is the convex hull of $\{(11,-1,-1,-1),(-1,-1,5,-1),(-1,5,-1,-1),(-1,-1,-1,-1)$, $(-1,-1,-1,1)\}$, a reflexive polytope in $\mathbb{R}^{4}$. The dual polytope $\Delta^{0}$ is the convex hull of $w_{0}=(-1,-2,-2,-6), w_{1}=(1,0,0,0), w_{2}=(0,1,0,0), w_{3}=(0,0,1,0)$ and $w_{4}=(0,0,0,1)$. Then, computations show that the only lattice point in the relative interior of a face $F^{0}$ of $\Delta^{0}$ with $1 \leq \operatorname{dim} F^{0} \leq 2$ is $(0,-1,-1,-3)$ and $F^{0}$ is the convex hull of $w_{0}$ and $w_{1}$. This shows that the dimension of the only twisted sector is 1, so that (3.1) implies (4.2) for generic anticanonical hypersurfaces of $\mathbb{P}(1,1,2,2,6)$.

Analogously, $\mathbb{P}(1,1,1,3,3)=\mathbb{P}_{\Delta}$ where $\Delta$ is the convex hull of $\{(8,-1,-1,-1)$, $(-1,-1,2,-1),(-1,2,-1,-1),(-1,-1,-1,-1),(-1,-1,-1,2)\}$, and the dual polytope $\Delta^{0}$ is the convex hull of $w_{0}=(-1,-1,-3,-3), w_{1}=(1,0,0,0), w_{2}=$ $(0,1,0,0), w_{3}=(0,0,1,0)$ and $w_{4}=(0,0,0,1)$. In this case, the only lattice point in the relative interior of a face $F^{0}$ of $\Delta^{0}$ with $1 \leq \operatorname{dim} F^{0} \leq 2$ is $(0,0,-1,-1)$ and $F^{0}$ is the convex hull of $w_{0}, w_{1}$ and $w_{2}$. Hence, if $X$ is a generic anticanonical hypersurface of $\mathbb{P}(1,1,1,3,3)$, it has a twisted sector $X_{t}$ isomorphic to $X \cap \overline{O_{\tau}}$, with $\operatorname{dim} \overline{O_{\tau}}=4-\left(1+\operatorname{dim} F^{0}\right)=1$, so $\operatorname{dim} X_{t}=0$, and, by (3.1), $i_{t} \neq i_{I(t)}$, so that (4.2) fails.

The same techniques can be applied further to test (4.2) in this toric setting. It would be very interesting to understand how common condition (4.2) is.

\section{Hodge structure}

In this section we will first construct polarized Hodge structures on the primitive part of $H_{o r b}^{k}(X)$ for appropriate orbifolds. Then we will see that for these orbifolds the full cohomology carries the structure described in Example 2.4. Finally, we use this last property to construct a polarized variation of Hodge structure associated to $X$.

In order to define a Hodge structure on $H_{o r b}^{k}(X)$ a first requirement is that the cohomology be integrally graded, which immediately leads to $X$ being an $S L$ orbifold. Also, we need $X$ to be projective satisfying condition (4.2), all conditions that we will assume in this section.

Let $X$ be a projective orbifold of dimension $n$ and $\omega \in H^{1,1}(X, \mathbb{R})$ a Kähler class. The primitive orbifold cohomology is defined by

$$
\left(H_{\text {orb }}^{p}\right)_{o}(X, \mathbb{C}):=\operatorname{ker}\left(L_{\omega}^{n-p+1}: H_{\text {orb }}^{p}(X, \mathbb{C}) \rightarrow H_{\text {orb }}^{2 n-p+2}(X, \mathbb{C})\right)
$$

where $L_{\omega}$ is defined by (4.1). For the Dolbeault groups we define

$$
\left(H_{\text {orb }}^{p, q}\right)_{o}(X):=H_{\text {orb }}^{p, q}(X) \cap\left(H_{\text {orb }}^{p+q}\right)_{o}(X, \mathbb{C}) .
$$

Theorem 5.1. Let $X$ be a projective SL-orbifold of dimension $n$ satisfying condition (4.2). Then, for each $k$ the decomposition

$$
H_{\text {orb }}^{k}(X, \mathbb{C})=\bigoplus_{p} H_{o r b}^{p, k-p}(X)
$$


is a Hodge structure of weight $k$ on $H_{\text {orb }}^{k}(X, \mathbb{R})$. Furthermore, for any Kähler class $\omega$, the decomposition

$$
\left(H_{\text {orb }}^{k}\right)_{o}(X, \mathbb{C})=\bigoplus_{p}\left(H_{o r b}^{p, k-p}\right)_{o}(X)
$$

defines a Hodge structure of weight $k$ that is polarized by the form $Q_{k}$, defined as the direct sum over all $t \in T$ of

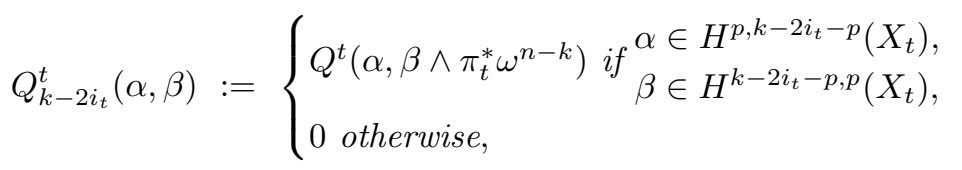

where

$$
Q^{t}(\alpha, \beta):=(-1)^{k(k-1) / 2+i_{t}} \int_{X_{t}} \alpha \wedge \beta \quad \text { for } \alpha \in H^{k-2 i_{t}}\left(X_{t}\right) .
$$

Proof. Since $\omega$ is a Kähler class on $X, \pi_{t}^{*} \omega$ is a Kähler class on the sector $X_{t}$. By Theorem 2.2, since each $X_{t}$ is a projective orbifold, each $H_{o}^{k-2 i_{t}}\left(X_{t}\right)$ has a Hodge structure of weight $k-2 i_{t}$ given by

$$
H_{o}^{k-2 i_{t}}\left(X_{t}, \mathbb{C}\right)=\bigoplus_{p}\left(H^{p, k-2 i_{t}-p}\left(X_{t}\right) \cap H_{o}^{k-2 i_{t}}\left(X_{t}\right)\right),
$$

and this structure is polarized by the form (5.3).

Tensoring the polarized Hodge structure defined above with $\mathbb{R}\left(i_{t}\right)$ (where $\mathbb{R}(k)$ is $\mathbb{R}$ viewed as a polarized Hodge structure of weight $2 k$ ) we obtain a new polarized Hodge structure of weight $k$ on $H_{o}^{k-2 i_{t}}\left(X_{t}\right)$ :

$$
H_{o}^{k-2 i_{t}}\left(X_{t}, \mathbb{C}\right)=\bigoplus_{p}\left(H^{p-i_{t}, k-p-i_{t}}\left(X_{t}\right) \cap H_{o}^{k-2 i_{t}}\left(X_{t}, \mathbb{C}\right)\right)
$$

This structure is still polarized by the same form $Q_{k-2 i_{t}}^{t}$.

Taking the direct sum over all sectors $t \in T$ the result follows.

Remark 5.2. The Hodge structure on $H_{\text {orb }}^{k}(X)$ is already implicit in 7 , Proposition 3.3.2]. An important point is that in order to define the polarization we are not using the Kodaira-Serre duality used in that paper. Instead, we are using integration on $X_{t}$ which is only possible under condition (4.2). This form, that we found naturally in the Hodge theoretic setting, was proposed in [11] in connection with the signature of the Poincaré form for the Kummer surface.

In Theorem 5.1 we considered the forms $Q^{t}$ defined by (5.4). We now define $Q$ to be the direct sum of the forms $Q^{t}$ over all $t \in T$. A routine check shows that this form is bilinear, nondegenerate and $(-1)^{n}$-symmetric.

Theorem 5.3. Let $X$ be a projective $S L$-orbifold of dimension $n$ satisfying the Hard Lefschetz Condition (4.2) and let $\omega$ be a Kähler class. Then the mixed Hodge structure $(W, F)$ defined by the bigrading $I^{p, q}:=H_{\text {orb }}^{n-q-p}(X)$ :

$$
W_{l}:=\bigoplus_{k \geq 2 n-l} H_{o r b}^{k}(X, \mathbb{C}), \quad F^{p}:=\bigoplus_{a} \bigoplus_{b \leq n-p} H_{o r b}^{a, b}(X)
$$

is a weight $n$ MHS polarized by the operator $L_{\omega}$ defined in (4.1) with the bilinear form $Q$ defined above. 
Proof. Since $\smile_{\text {orb }}$ preserves the bigrading of the Dolbeault groups, $L_{\omega}$ is a $(-1,-1)$ morphism of $(W, F)$ and, in particular, $L_{\omega}^{n+1}=0$. To check that $L_{\omega} \in \mathfrak{g}_{\mathbb{R}}$, it suffices to check that

$$
Q^{t}\left(L_{\omega} \alpha, \beta\right)+Q^{t}\left(\alpha, L_{\omega} \beta\right)=0 \text { for } \alpha, \beta \in H^{*}\left(X_{t}\right)
$$

since the decomposition in sectors is $Q$-orthogonal, and this is a straightforward computation. The reality of $L_{\omega}$ follows from $\omega$ being real and the reality of $\smile_{\text {orb }}$.

To show that $W$ defined by (5.5) is $W\left(L_{\omega}\right)[-n]$ it suffices to check that $L_{\omega}\left(W_{l}\right) \subset$ $W_{l-2}$ for all $l$ and that $L_{\omega}^{j}: W_{j+n} / W_{j+n-1} \rightarrow W_{-j+n} / W_{-j+n-1}$ are isomorphisms for all $j$. The first condition follows immediately from the definitions. The second condition is equivalent to requiring that $L_{\omega}^{j}: H_{o r b}^{n-j}(X) \rightarrow H_{o r b}^{n+j}(X)$ be isomorphisms, which is true by Proposition 4.1 .

Note that, for each $t \in T$,

$$
Q^{t}\left(H^{a-i_{t}, b-i_{t}}\left(X_{t}\right), H^{c-i_{t}, d-i_{t}}\left(X_{t}\right)\right)=0
$$

unless $a+c=n$ which, by the $Q$ orthogonality of the sector decomposition, implies that $Q\left(H_{\text {orb }}^{a, b}(X), H_{\text {orb }}^{c, d}(X)\right)=0$ if $a+c \leq n-1$. In turn, this last condition implies that $Q\left(F^{p}, F^{n-p+1}\right)=0$ for all $p$.

Finally we have to prove that the Hodge structure of weight $n+l$ induced by $F$ on $\operatorname{ker}\left(L_{\omega}^{l+1}: \mathrm{Gr}_{n+l}^{W} \rightarrow \mathrm{Gr}_{n-l-2}^{W}\right)$ is polarized by $Q\left(\cdot, L_{\omega}^{l} \cdot\right)$. Using (5.5) we are studying the Hodge structure on $\left(H_{o r b}^{n-l}\right)_{o}(X)$ given by (5.2) (with $k=n-l$ ) and the polarization is precisely the one that, because of Theorem [5.1 polarizes this structure.

A notion of Frobenius module is introduced in [2] for $V=\bigoplus_{p=0}^{n} V_{2 p}$ a finite dimensional, graded, $\mathbb{C}$-vector space with a $\operatorname{Sym} V_{2}$-module structure. A natural generalization exists if $V$ is a Sym $W$-module for a subspace $W \subset V_{2}$. In this context, Theorem 5.3 implies that $V:=\bigoplus_{p=0}^{n} H_{o r b}^{p, p}(X)$ is a polarizable $\operatorname{Sym} H^{1,1}(X)$ Frobenius module of weight $n:=\operatorname{dim}_{\mathbb{C}} X$.

An immediate consequence of Theorems 5.3 and (2.3) in [4] is the following.

Corollary 5.4. Let $X$ be a projective $S L$-orbifold of dimension $n$ satisfying condition (4.2). Given $\omega_{1}, \ldots, \omega_{r}$ in the Kähler cone of $X$ such that they form a basis of $H^{1,1}(X)$ and $F, Q$ as in Theorem 5.3 , then

$$
\theta: U^{r} \rightarrow D, \quad \theta\left(z_{1}, \ldots, z_{r}\right):=\exp \left(\sum_{j=1}^{r} z_{j} L_{\omega_{j}}\right) \cdot F
$$

is a nilpotent orbit, where $U$ is the upper half plane $\operatorname{Im} z>0$. Moreover, if $\mathcal{K}_{\mathbb{C}}$ is the complexified Kähler cone of $X, \xi \mapsto \exp \left(L_{\xi}\right) \cdot F$ is a polarized variation of Hodge structure of weight $n$ defined over $\mathcal{K}_{\mathbb{C}}$.

Proof. Since $\smile_{\text {orb }}$ is commutative for multiplication by 2-classes, $\left\{L_{\omega_{1}}, \ldots, L_{\omega_{r}}\right\}$ is a commuting subset of $\mathfrak{g}_{\mathbb{R}}$. By Theorem 5.3 the weight filtration $W\left(\sum_{j=1}^{r} \lambda_{j} L_{\omega_{j}}\right)$ is the constant $W$ defined by (5.5), hence independent of $\lambda_{j}>0$. Again by the same result, $(W, F)$ is polarized by every $\sum_{j=1}^{r} \lambda_{j} L_{\omega_{j}}$ with $\lambda_{j}>0$. Noticing that the mixed Hodge structure $(W, F)$ is split over $\mathbb{R}$, Theorem (2.3) in 4 completes the proof. 


\section{REFERENCES}

1. W. L. Baily, On the imbedding of $V$-manifolds in projective space, Amer. J. Math. 79 (1957), 403-430. MR0100104 (20:6538)

2. E. Cattani and J. Fernandez, Frobenius modules and Hodge asymptotics, Comm. Math. Phys. 238 (2003), no. 3, 489-504. Also, arXiv:math.AG/0207279. MR1993382 (2004i:32022)

3. E. Cattani and A. Kaplan, Polarized mixed Hodge structures and the local monodromy of a variation of Hodge structure, Invent. Math. 67 (1982), no. 1, 101-115. MR0664326 (84a:32046)

4. - Degenerating variations of Hodge structure, Astérisque (1989), no. 179-180, 9, 67-96, Actes du Colloque de Théorie de Hodge (Luminy, 1987). MR 1042802 (91k:32019)

5. E. Cattani, A. Kaplan, and W. Schmid, Degeneration of Hodge structures, Annals of Mathematics 123 (1986), 457-535. MR0840721 (88a:32029)

6. W. Chen and Y. Ruan, Orbifold Gromov-Witten theory, Orbifolds in mathematics and physics (Madison, WI, 2001), Contemp. Math., vol. 310, Amer. Math. Soc., Providence, RI, 2002, pp. 25-85. Also, arXiv:math.AG/0103156. MR/1950939 (2003g:00020)

7. _ A new cohomology theory of orbifold, Comm. Math. Phys. 248 (2004), no. 1, 1-31. Also, arXiv:math.AG/0004129. MR2104605 (2005j:57036)

8. D. A. Cox and S. Katz, Mirror symmetry and algebraic geometry, American Mathematical Society, Providence, RI, 1999. MR1677117 (2000d:14048)

9. P. Griffiths (ed.), Topics in transcendental algebraic geometry, Annals of Mathematics Studies, vol. 106, Princeton, NJ, Princeton University Press, 1984. MR0756842 (86b:14004)

10. M. Poddar, Orbifold hodge numbers of Calabi-Yau hypersurfaces, Pacific J. Math. 208 (2003), no. 1, 151-167. Also, arXiv:math.AG/0107152. MR.1979377(2004k:32042)

11. Y. Ruan, Cohomology ring of crepant resolutions of orbifolds, arXiv:math.AG/0108195, January 2002.

12. - Stringy geometry and topology of orbifolds, Symposium in Honor of C. H. Clemens (Salt Lake City, UT, 2000), Contemp. Math., vol. 312, Amer. Math. Soc., Providence, RI, 2002, pp. 187-233. MR1941583 (2004b:32051)

13. M. Saito, Mixed Hodge modules, Publ. Res. Inst. Math. Sci. 26 (1990), no. 2, 221-333. MR 1047415 (91m:14014)

14. I. Satake, On a generalization of the notion of manifold, Proc. Nat. Acad. Sci. U.S.A. 42 (1956), 359-363. MR0079769 (18:144a)

15. W. Schmid, Variation of Hodge structure: the singularities of the period mapping, Invent. Math. 22 (1973), 211-319. MR0382272 (52:3157)

16. J. H. M. Steenbrink, Mixed Hodge structure on the vanishing cohomology, Real and complex singularities (Proc. Ninth Nordic Summer School/NAVF Sympos. Math., Oslo, 1976), Sijthoff and Noordhoff, Alphen aan den Rijn, 1977, pp. 525-563. MR0485870(58:5670)

Department of Mathematics, University of Utah, Salt Lake City, Utah 84112-0090 Current address: Instituto Balseiro, Univerisdad Nacional de Cuyo - C.N.E.A., Bariloche, Río Negro, R8402AGP, República Argentina

E-mail address: jfernand@ib.edu.ar 